ANNALES

POLONICI MATHEMATICI

XII (1962)

\title{
Sur un théorème de la géométrie différentielle globale
}

\author{
par S. GoEAB (Krak6w)
}

Etant donné dans l'espace euclidien $R_{3}$ une surface $S$ fermée orientable de classe $C^{2}$ on a, en désignant par $\mathcal{X}$ la courbure de Gauss, la formule

$$
\iint_{S} \chi d S=4 \pi(1-p),
$$

où $p$ est le genre de $\$$. Pour les surfaces qui sont topologiquement équivalentes à la sphère on a $p=0$ et la formule précédente se réduit à la suivante:

$$
\iint_{S} x d S=4 \pi
$$

Si nous désignons par $\mathcal{H}$ la courbure moyenne de $S$, alors, comme l'a montré H. Minkowski, l'intégrale

$$
\iint_{S} \mathcal{H} d \mathcal{S}
$$

représente, sous une hypothèse convenable concernant le signe, à un facteur constant près, la distance moyenne des plans tangents à $S$ à un point situé à l'intérieur de $\mathbb{S}$. Si le diamètre de $\mathbb{S}$ augmente à l'infini, alors l'intégrale (3) se comporte de façón analogue, ce qui peut être vérifié par l'exemple d'une sphère. $\Pi$ n'existe, par conséquent, aucune borne supérieure fixe pour l'intégrale (3). On peut cependant chercher une majoration de l'intégrale (3) au moyen du diamètre de $S$. En plaçant l'origine des coordonnées $O$ an milieu du segment réalisant le diamètre $D$ on obtient facilement que la distance de tout point $P$ de $S$ au point $O$ est au plus égale à $D \cdot \sqrt{3} / 2$, donc la distance de $O$ au plan tangent à $S$ au point $P$ est $\leqslant D \cdot \sqrt{3} / 2$. La distance moyenne $M$ vérifie aussi l'inégalité $M \leqslant D \cdot \sqrt{3} / 2$ et, par conséquent, nous avons

$$
\iint_{S} \mathcal{H} d S=4 \pi M \leqslant 4 \pi D \cdot \sqrt{3} / 2=2 \pi \sqrt{3} D .
$$

Bien entendu, cette évaluation n'est pas exacte. 
En désignant par $x_{1}, x_{2}$ les courbures (algébriques) des sections normales suivant les directions principales, on a les formules

$$
X=x_{1} \cdot x_{2}, \quad \mathcal{H}=\left(x_{1}+x_{2}\right) / 2 .
$$

La question se pose d'évaluer les intégrales

$$
I_{i}=\iint_{S} x_{i} d S \quad(i=1,2)
$$

Cependant une difficulté fondamentale consiste à donner pour les courbures $x_{i}$ une définition univoque, présentant un caractère intrinsèque et non accidentel. La définition

$$
x_{1}=\min \left(x_{1}, x_{2}\right), \quad x_{2}=\max \left(x_{1}, x_{2}\right),
$$

la plus simple du point de vue analytique, ne serait pas utile ici. La détermination du numérotage des $x_{1}, x_{2}$ en un point fixé qui n'est pas un ombilic peut être prolongée d'une façon univoque seulement sur un domaine qui ne contient aucun ombilic, donc pas à toute la surface fermée $S$ qui contient certainement des ombilics.

Tenant compte de ces difficultés de détermination des fonctions $x_{1}(P), x_{2}(P)$ sur toute la surface fermée $S$, nous nous occuperons dans ce travail des surfaces fermées de révolution de genre zéro pour lesquelles la définition univoque des fonctions $x_{1}$ et $x_{2}$ ne présente aucune difficulté. Nous adoptons notamment la définition suivante:

$$
\begin{aligned}
& x_{1} \stackrel{d f}{=} \text { courbure normale dans le sens du parallèle, } \\
& x_{2} \stackrel{\text { df }}{=} \text { courbure normale dans le sens du méridien. }
\end{aligned}
$$

La définition (8) ne se confond pas en général avec la définition (7).

$\Pi$ est bien connu que le signe de la courbure moyenne $\mathcal{H}$ (contrairement à $\chi$ ) dépend de l'orientation de la surface $S$. Nous établirons une paramétrisation de $S$ telle que le vecteur normal $N$ soit dirigé à l'intérieur de la surface $S$. Dans ce but nous prendrons l'axe des $x_{3}$ pour axe de révolution. L'hypothèse que la surface $S$ est de genre zéro implique que le méridien est un arc simple de Jordan, dont les extrémités sont situées sur l'axe de révolution. Pour premier paramètre $u$ nous prenons la longeur d'arc du méridien comptée à partir du pôle méridional, le secod paramètre $v$ sera la longitude et le méridien nul sera celui qui est contenu dans le demi-plan $x_{1}, x_{3}\left(x_{1} \geqslant 0\right)$. Bien entendu, l'angle $v$ est compté conformément à l'orientation du plan $\left(x_{1}, x_{2}\right)$. Dans ces hypothèses les équations paramétriques de notre surface $S$ peuvent être mises sous la forme

$$
x_{1}=\varphi(u) \cos v, \quad x_{2}=\varphi(u) \sin v, \quad x_{3}=\psi(u)
$$


où, en désignant par $L$ la longueur du méridien, on a les relations suivantes

$$
\begin{gathered}
\varphi(0)=\varphi(L)=0, \\
\varphi^{\prime 2}(u)+\psi^{\prime 2}(u) \equiv \mathbf{1}, \\
\varphi^{\prime}(\mathbf{0})=\mathbf{1}, \quad \varphi^{\prime}(L)=-\mathbf{1}, \\
\psi^{\prime}(\mathbf{0})=\psi^{\prime}(L)=\mathbf{0}, \\
\psi(L)>\psi(0) .
\end{gathered}
$$

Les relations (12) et (13) découlent de l'hypothèse que $S$ admet un plan tangent aussi aux pôles, nécessairement perpendiculaire à l'axe de révolution. Comme les fonctions $\varphi$ et $\psi$ ne dépendent que de la variable $u$, nous omettrons dans la suite le symbole de la variable indépendante. Les courbures $\mathcal{X}$ et $\mathcal{H}$ s'expriment (sauf aux pôles) par les formules

$$
\begin{gathered}
\mathcal{X}=\frac{\psi^{\prime}\left(\varphi^{\prime} \psi^{\prime \prime}-\psi^{\prime} \varphi^{\prime \prime}\right)}{\varphi}=\frac{\psi^{\prime} \Delta}{\varphi}, \\
\mathcal{H}=\frac{\psi^{\prime}+\varphi\left(\varphi^{\prime} \psi^{\prime \prime}-\psi^{\prime} \varphi^{\prime \prime}\right)}{2 \varphi}=\frac{\psi^{\prime}+\varphi \Delta}{2 \varphi}, \\
\Delta=\varphi^{\prime} \psi^{\prime \prime}-\psi^{\prime} \varphi^{\prime \prime} .
\end{gathered}
$$

Remarquons que $\Delta$ représente la courbure (algébrique) du méridien. Les courbures principales $x_{1}, x_{2}$ sont racines de l'équation

$$
x^{2}-2 \nVdash x+X=0 \text {. }
$$

En tenant compte des relations (15) et (16) nous obtenons pour $x$

$$
x=\frac{\psi^{\prime}+\varphi \Delta+\varepsilon\left(\psi^{\prime}-\varphi \Delta\right)}{2 \varphi}, \quad \varepsilon= \pm 1,
$$

donc les deux valeurs suivantes

$$
\psi^{\prime} / \varphi, \quad \Delta .
$$

Comme $\Delta$ est la courbure du méridien, nous obtenons conformément à la définition

$$
\varkappa_{1}=\psi^{\prime} / \varphi, \quad \varkappa_{2}=\Delta
$$

Mais

$$
d S=\sqrt{g_{11} g_{22}-g_{12}^{2}} d u d v=\sqrt{\varphi^{2}} d u d v=\varphi d u d v .
$$

Nous avons supposé que le méridien nul, d'équations

$$
x_{1}=\varphi(u), \quad x_{3}=\psi(u),
$$

est situé du côté positif de l'axe des $x_{1}$, donc

$$
\varphi(u) \geqslant 0 \text {, }
$$


Nous avons, par conséquent,

$$
\begin{gathered}
I_{1}=\iint_{S} x_{1} d S=\iint_{R} \psi^{\prime}(u) d u d v, \\
I_{2}=\iint_{S} x_{2} d S=\iint_{R} \Delta(u) \varphi(u) d u d v,
\end{gathered}
$$

où $R$ est le rectangle défini par les inégalités

$$
0 \leqslant u \leqslant L, \quad 0 \leqslant v \leqslant 2 \pi .
$$

L'intégrale (23) est facile à calculer. Nous avons notamment

$$
\iint_{\boldsymbol{R}} \psi^{\prime}(u) d u d v=\int_{0}^{2 \pi} d v \int_{0}^{L} \psi^{\prime}(u) d u=2 \pi \int_{0}^{L} \psi^{\prime}(u) d u=2 \pi[\psi(L)-\psi(0)] .
$$

En désigant par $h$ la distance des pôles ou la largeur de $S$ dans le sens de l'axe de révolution nous avons

$$
h=\psi(L)-\psi(0)
$$

et on obtient finalement pour $I_{1}$ la simple formule suivante:

$$
I_{1}=2 \pi h \text {. }
$$

L'intégrale $I_{1}$ est donc proportionnelle à la distance des pôles et toujours positive.

Pour l'intégrale $I_{1}$ nous allons trouver des bornes supérieure et inférieare. Nous avons

$$
I_{2}=2 \pi \int_{0}^{L} \varphi(u) \Delta(u) d u
$$

et la valeur $I_{2}$ peut être négative, puisque $\varphi \geqslant 0$, mais $\Delta$ représente une quantité algébrique.

Si nous supposons que $S$ est une surface convexe, le méridien sera aussi une courbe convexe et nous aurons

$$
\Delta \geqslant 0 \text {. }
$$

Dans ce cas l'intégrale $I_{2}$ sera positive. Nous trouverons sa borne supérieure en désignant par s la largeur de la section axiale de $S$, c'est-à-dire le diamètre du cylindre vertical circonscrit à $S$. Dans ce cas nous avons

$$
s / 2=\max \varphi(u)
$$

et, comme $\varphi \geqslant 0, \Delta \geqslant 0$, on pent écrire

$$
I_{2}=2 \pi \int_{0}^{L} \varphi \Delta d u \leqslant 2 \pi \int_{0}^{L} \Delta \cdot \max \varphi d u=\pi s \int_{0}^{L} \Delta(u) d u .
$$


Mais d'autre part, on a

$$
\Delta=d \Theta / d u \text {, }
$$

si $\Theta$ est l'angle entre la tangente orientée du méridien et l'axe des $x_{1}$. En égard à la convexité du méridien on a

$$
\int_{0}^{L} \Delta(u) d u=\int_{0}^{L} \frac{d \Theta}{d u} d u=\Theta(L)-\Theta(0)=\pi,
$$

d'où l'on tire l'évaluation suivante

$$
I_{2} \leqslant \pi^{2} s \text {. }
$$

L'inégalité précédente peut être remplacée par une inégalité forte puisque le méridien est de classe $C^{2}$ et, par conséquent, il existe des valeurs $u<u_{0}\left(\varphi\left(u_{0}\right)=s / 2\right)$ pour lesquelles $\Delta>0$. Nous avons donc

$$
I_{2}<\pi^{2} 8 \text {. }
$$

Cette évaluation est inexacte pour la sphère. Pour la sphère on a notamment

$$
I_{2}=4 R \pi
$$

et l'erreur est $57 \%$. Cette évaluation est, d'autre part, exacte en ce sens que pour tout nombre positif $\varepsilon$ il existe une surface de révolution telle que

$$
I_{2}=\pi^{2} s-\varepsilon .
$$

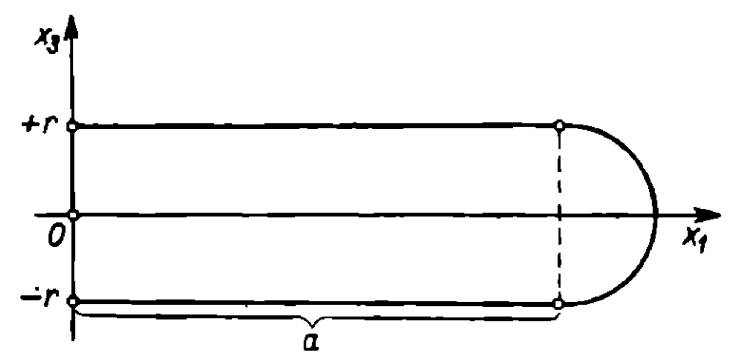

Fig. 1

Nous allons construire un exemple d'une telle surface dont le méridien est de classe $C^{2}$ par morceaux ( $\Delta$ aura deux discontinuités; ce fait ne jouera ici aucun rôle, car il s'agit de l'intégrale dont un des facteurs sous le signe de l'intégrale est 4 ). Supposons le méridien composé de deux segments de longueur $a$, parallèles à l'axe des $x_{1}$ et de la demi-circonférence de diamètre $2 r=h$, comme le montre la figure 1. Alors

$$
\int_{0}^{L} \varphi \Delta d u=\int_{0}^{a} \varphi \Delta d u+\int_{a}^{a+\pi} \varphi \Delta d u+\int_{a+\pi}^{2 a+r \pi} \varphi \Delta d u .
$$


La première et troisième intégrale du second membre sont nulles, car $\Delta=0$ dans les intervalles correspondants. $I 1$ reste donc

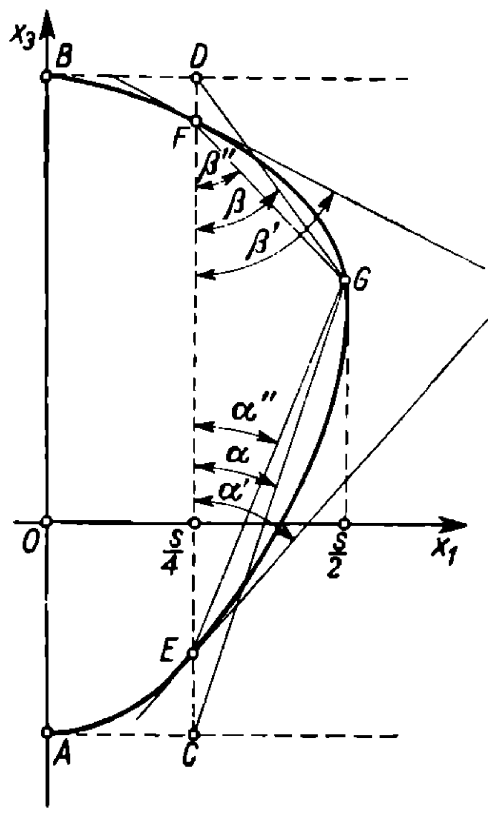

Fig. 2

$$
\int_{a}^{a+r \pi} \varphi \Delta d u=\frac{1}{r} \int_{a}^{a+r \pi} \varphi d u
$$

Cette dernière intégrale est facile à calculer. Elle est égale à $a \pi r+2 r^{2}$. Nous avons ainsi

$$
\begin{aligned}
I_{2} & =2 \pi \int_{0}^{L} \varphi \Delta d u=2 \pi(a \pi+2 r) \\
& =\pi^{2}(s-2 r)+4 \pi r .
\end{aligned}
$$

$\Pi$ est évident que $a$ étant fixé, si nous faisons tendre $r$ vers zéro nous aurons

$$
I_{2} \rightarrow \pi^{2} s,
$$

ce qui démontre notre conclusion.

Passons au calcul qui nous permettra d'obtenir la borne inférieure de l'intégrale $I_{2}$. Désignons, comme auparavant, par $u_{0}$ la valeur pour laquelle on a

et soit

$$
\varphi\left(u_{0}\right)=s / 2=\max \varphi(u)
$$

\section{Comme}

$$
\mu \stackrel{\mathrm{df}}{=} \frac{\psi\left(u_{0}\right)-\psi(0)}{\psi(L)-\psi(0)}
$$

nous avons

$$
\psi(0)<\psi\left(u_{0}\right)<\psi(L)
$$

$$
0<\mu<1
$$

Considérons la figure 2; traçons dans le plan $\left(x_{1}, x_{3}\right)$ la droite $x_{1}=s / 4$ et désignons par $A, B, C, D, G$ respectivement les points $A[0, \psi(0)]$, $B[0, \psi(L)], \quad C[s / 4, \psi(0)], D[s / 4, \psi(L)], G\left[s / 2, \psi\left(u_{0}\right)\right]$. La droite $x_{1}=s / 4$ coupera le méridien convexe en deux points $E\left[s / 4, \psi\left(u_{1}\right)\right], F\left[s / 4, \psi\left(u_{2}\right)\right]$, où $u_{1}<u_{0}<u_{2}$. Désignons ensuite par $a, \beta$ les angles entre les vecteurs $\overrightarrow{C G}, \overrightarrow{C D}$ resp. $\overrightarrow{D G}, \overrightarrow{D C}$. Désignons enfin par $\alpha^{\prime}, \beta^{\prime}$ respectivement les angles contenus entre les tangentes au méridien aux points $E$ resp. $F$ et la droite $C D$, et par $\alpha^{\prime \prime}, \beta^{\prime \prime}$ les angles analogues entre les vecteurs $\overrightarrow{E G}, \overrightarrow{E D}$ et $\overrightarrow{F G}, \overrightarrow{F C}$. Des considérations géométriques élémentaires donnent les inégalités

$$
a^{\prime \prime}>a, \quad \beta^{\prime \prime}>\beta .
$$


Le méridien étant convexe, on conclut

Par conséquent

$$
\alpha^{\prime}>a^{\prime \prime}, \quad \beta^{\prime}>\beta^{\prime \prime} \text {. }
$$

$$
\alpha^{\prime}>a, \quad \beta^{\prime}>\beta .
$$

La variation totale de l'angle de la tangente au méridien le long de l'arc $\widetilde{E F}$ est égale à $a^{\prime}+\beta^{\prime}$. Cette variation est d'autre part égale à

$$
\alpha^{\prime}+\beta^{\prime}=\int_{u_{1}}^{u_{2}} \Delta d u .
$$

Des inégalités (34) on tire

$$
\int_{u_{1}}^{u_{2}} \Delta d u>\alpha+\beta .
$$

Mais

$$
\int_{0}^{L} \varphi \Delta d u>\int_{u_{1}}^{u_{2}} \varphi \Delta d u .
$$

Toutes les abscisses des points de l'arc $\widetilde{E F}$ étant au moins égales à $s / 4$ on peut écrire

$$
u_{1} \leqslant u \leqslant u_{2} \Rightarrow \varphi(u) \geqslant s / 4
$$

d'où résulte l'inégalité

$$
\int_{0}^{L} \varphi \Delta d u>\frac{8}{4} \int_{u_{1}}^{u_{2}} \Delta d u .
$$

Les inégalités (36) et (37) donnent

$$
\int_{0}^{L} \varphi \Delta d u>\frac{8}{4}(\alpha+\beta) .
$$

Nous avons ensuite

$$
\alpha=\operatorname{arctg} \frac{s}{4 \mu h}, \quad \beta=\operatorname{arctg} \frac{s}{4 h(1-\mu)} .
$$

Il est facile de montrer que la fonction

$$
\operatorname{arctg} \frac{s}{4 h \mu}+\operatorname{arctg} \frac{s}{4 h(1-\mu)},
$$

où $s, h$ sont considérés comme des constantes et $\mu$ comme une variable parcourant l'intervalle $(0,1)$, atteint son minimum pour $\mu=\frac{1}{2}$. On a, par conséquent,

$$
\operatorname{arctg} \frac{8}{4 h \mu}+\operatorname{arctg} \frac{s}{4 h(1-\mu)}>2 \operatorname{arctg} \frac{s}{2 h}
$$


Done

$$
I_{2}>\pi s \operatorname{arctg} \frac{s}{2 h}
$$

De l'inégalité connue

$$
2 \operatorname{arctg} \frac{x}{2}>\operatorname{arctg} x \quad \text { pour } \quad x>0
$$

on obtient ensuite

$$
I_{2}>\frac{\pi}{2} s \operatorname{arctg} \frac{s}{h}
$$

En résumant les résultats obtenus nous pouvons énoncer les deux théorèmes suivants.

THónonìme 1. Si la surface $S$ de genre zéro et de classe de régularité $C^{2}$ est une surface de révolution, on a la formule

$$
I_{1}=2 \pi h
$$

THÉonk̀me 2. Si la surface $S$ de genre zéro et de classe de régularité $C^{2}$ est une surface convexe de révolution, on a les inégalités suivantes

$$
\frac{\pi s}{2} \operatorname{arctg} \frac{s}{h}<I_{2}<\pi^{2} s
$$

Dans ces formules interviennent deux constantes $h, s$ liées à la forme géométrique de la surface $S$. Nous rappelons que $h$ est la largeur de $S$ dans le sens vertical alors que $s$ est sa largeur dans le sens horizontal.

Pour 8 petits on a

$$
\operatorname{arctg} \frac{8}{h} \approx \frac{8}{h}
$$

et la borne inférieure est donnée par la quantité

$$
\frac{\pi s}{2} \operatorname{arctg} \frac{s}{h} \approx \frac{\pi}{2 h} 8^{2}=\frac{\pi}{2} \cdot s \cdot \frac{s}{h}
$$

qui est un infiniment petit du deuxième ordre. L'exemple cité ci dessous nous montre que $I_{2}$ peut être un infiniment petit d'ordre plus grand que 1 par rapport à 8 .

Calculons $I_{2}$ pour un ellipsoïde de révolution. Si nous posons

$$
h=2 b, \quad s=2 a \quad(a, b \text { demi-axes de l'ellipse })
$$

alors (l'intégrale $I_{2}$ peut être calculée effectivement bien que le calcul soit différent suivant que $\lambda=b / a$ est plus grand ou plus petit que 1) nous obtenons 


$$
I_{2}=\left\{\begin{array}{lll}
\frac{4 \pi a^{2}}{\sqrt{a^{2}-b^{2}}} \operatorname{arctg} \sqrt{\frac{1}{\lambda^{2}}-1} & \text { pour } & \lambda<1, \\
\frac{4 \pi a^{2}}{\sqrt{b^{2}-a^{2}}} \log \left[\lambda+\sqrt{\lambda^{2}-1}\right] & \text { pour } & \lambda>1 .
\end{array}\right.
$$

Si l'ellipsoïde est de forme aplatie ( $s / h$ grand), alors $\lambda$ est petit. Réciproquement, si l'ellipsoïde est en forme d'aiguille ( $s / h$ petit), alors $\lambda$ est grand.

Dans le premier cas nous avons

$$
\frac{a^{2}}{\sqrt{a^{2}-b^{2}}} \operatorname{arctg} \sqrt{\frac{1-\lambda^{2}}{\lambda^{2}}}=\frac{s}{2 \sqrt{1-\lambda^{2}}} \operatorname{arctg} \frac{\sqrt{1-\lambda^{2}}}{\lambda} \approx \frac{8}{2} \cdot \frac{\pi}{2}=\frac{\pi s}{4}
$$

et, par conséquent,

Dans le deuxième cas

$$
I_{2} \approx \pi^{2} s
$$

$$
\frac{a^{2}}{\sqrt{b^{2}-a^{2}}} \log \left[\lambda+\sqrt{\lambda^{2}-1}\right] .=\frac{s}{2 \sqrt{\lambda^{2}-1}} \log \left|\lambda+\sqrt{\lambda^{2}-1}\right| .
$$

Ici, quand $\lambda$ tend vers l'infini,

$$
\frac{\log \left(\lambda+\sqrt{\lambda^{2}-1}\right)}{\sqrt{\lambda^{2}-1}} \rightarrow 0
$$

donc $I_{2} \rightarrow 0$ et dans ce cas la borne inférieure fournit une meilleure évaluation.

Remarque. L'hypothèse que le genre de $S$ est zéro est essentielle dans les théorèmes énoncés ci-dessus. Pour les surfaces de genre un les évaluations précédentes ne sont plus valables, comme le montre l'exemple d'un tore de révolution pour lequel on a

$$
I_{1}=0, \quad I_{2}=4 a \pi,
$$

où $a$ désigne la distance du centre cercle-méridien à l'axe de révolution. 